\title{
The Effect of Imperfect Preventive Maintenance on Component and System Reliability
}

\author{
Shawulu Hunira Nggada \\ Department of Computer Science \\ School of Computing and Informatics, Polytechnic of Namibia \\ 13 Storch Street, Windhoek, Namibia \\ snggada@polytechnic.edu.na
}

\begin{abstract}
The improvement of system reliability can be achieved by improving the reliabilities of its constituent components. This could be done through maintenance actions that are performed on the components. There are two extreme states in which a component could assume following a maintenance stage; bad-as-old (BAO) or good-as-new (GAN). For some components of the system, the maintenance actions may bring them to an in-between state. This in-between state is achieved under the assumptions of imperfect maintenance. This paper investigates the improvement in reliability of a component and system in the in-between state through imperfect preventive maintenance policy. Thus, it establishes a mathematical model for component reliability evaluation under assumptions of imperfect preventive maintenance.
\end{abstract}

Keywords: Reliability, preventive maintenance, improvement factor

\section{Introduction}

Preventive maintenance (PM) activities can minimise breakdowns (or failures) and improve the overall reliability of a system $[1,2]$. The number and type of maintenance activities earmarked upon for a component depends on its design characteristics. The following are possible maintenance activities that may be performed on a component; lubricating, cleaning, tightening, adjusting, topping and simple repair (e.g., replacement of seals and rings). Simple repair is also referred to as minimal repair [3, 4], and this name is adopted in this paper. The time at which maintenance actions are performed on a component is referred to as maintenance stage. Under PM policy, each maintenance stage of a given component is a periodic interval. There are two extreme states or conditions for which a component can assume after a given PM stage assuming maintenance actions follow prescribed procedures and are performed by experts. These are bad-as-old (BAO) and goodas-new (GAN). In the former state, the maintenance actions have no effect on the component while in the latter the maintenance actions renew the component. Maintenance actions may however improve the condition of a component to an in-between state; which lies between BAO and GAN. Such maintenance policy is termed as imperfect preventive maintenance (IPM).

According to the proportional age reduction (PAR) model, the age of a component after a maintenance stage depends on the effectiveness of the maintenance actions [3, 5]. This effectiveness is what is termed as improvement factor [6,7]. The age that a component assumes after maintenance stage is known as effective age. This implies that the new effective age of the component lies in-between its effective age following the previous 
maintenance stage and the age at current maintenance stage. Using the PAR model, the effective age of a component at the $\mathrm{j}$-th maintenance stage is modeled as in equation $1[6,7]$.

$W_{j}^{+}=\left(1-f_{j}\right) j T_{p}$

Where: $j$ is the current count of the PM stage

$W_{j}^{+}$is the effective age at the $j$-th PM stage

$f_{j}$ is the improvement factor at the $j$-th PM stage

$T_{p}$ is the component's regular interval for maintenance referred to as PM interval

It is trivial from equation 1 that when the improvement factor $f=1$, the component is renewed and the maintenance actions result into GAN also referred to as perfect preventive maintenance. Similarly when $f=0$, the maintenance actions have no effect on the age of the component and the effective age of the component is same as its age at the PM stage. Under IPM policy, the improvement factor $f$ is less than 1, i.e. $0 \leq \mathrm{f}<1$.

Although several works on maintenance exist, maintenance modelling is quite underdeveloped [5]. According to Sanchez et al. [7], "in many cases there is limited knowledge on the proper model to represent a problem, and thus results in that for a particular imperfect maintenance model, there are multiple competing models producing different approximation of the same problem." This paper contributes to addressing maintenance modeling of reliability under the assumptions of imperfect preventive maintenance using the proportional age reduction model. It also assumes that improvement factor is constant and that component failure follows the Weibull distribution.

In Section 2, the general concept behind modelling reliability under maintenance as established in literature is presented. This is utilised in establishing a universal model for component reliability under IPM policy. A modelling of Weibull component reliability model which is based on the universal reliability model is then presented. Section 3 discusses the case study on which the established reliability model is evaluated. Section 4 presents evaluations and results of the IPM reliability model on the case study. Conclusions are drawn in Section 5.

\section{Reliability Modeling under Preventive Maintenance}

To model the reliability of a component under preventive maintenance, the following two scenarios are considered $[4,8]$.

(i) The probability of surviving until the $j$-th $\mathrm{PM}$ stage; i.e. PM time being $j T_{p}$

(ii) The probability of surviving the remaining time $t-j T_{p} ; j T_{p} \leq t \leq \theta$

Where: $j$ is same as describe in the previous section; $j$-th PM stage

$t$ is the calendar age of the component

$\theta$ is the useful life of the component or the scale of time under consideration

To model the reliability of the component, it is essential to know its total number of PM stages $n$. This can be calculated using equation 2 .

$n= \begin{cases}Q\left(\frac{M T B F}{T_{p}}\right) & ; M T B F \leq R T \\ Q\left(\frac{R T}{T_{p}}\right) & ; M T B F>R T\end{cases}$ 
Where $M T B F$ is the mean time between failures of the component, $R T$ is the useful system operational life time also known as system risk time and $Q$ is the integer quotient of the division.

The reliability of a component on the $j$-th PM stage can be constructed as shown in equation 3 [4].

$R_{j}(t)=R_{0 j} R_{v j}(t)$

Where $\mathrm{R}_{\mathrm{oj}}$ is the probability of surviving until the $j$-th PM stage, and $R_{v j}$ is the probability of surviving the remaining time.

\subsection{Universal Reliability Modeling of IPM}

To model the universal reliability of a component under IPM, Figure 1 is considered. It shows the PM stages of a component from when $j=1$ to $j=n$. For a simple mathematical notation with respect to equation 1 , let $t_{r}=(1-f) T_{p}$; under constant improvement factor. At any PM stage where $t_{r e m}=0$, the probability of surviving the $j$-th PM stage is modeled as follows.

$$
\begin{array}{ll}
R(t)=R\left(T_{p}\right) & ; j=1 \wedge t=T_{p} \\
R(t)=R\left(T_{p}\right) R\left(t_{r}, t_{r}+T_{p}\right) & ; j=2 \wedge t=2 T_{p} \\
R(t)=R\left(T_{p}\right) R\left(t_{r}, t_{r}+T_{p}\right) R\left(2 t_{r}, 2 t_{r}+T_{p}\right) & ; j=3 \wedge t=3 T_{p}
\end{array}
$$

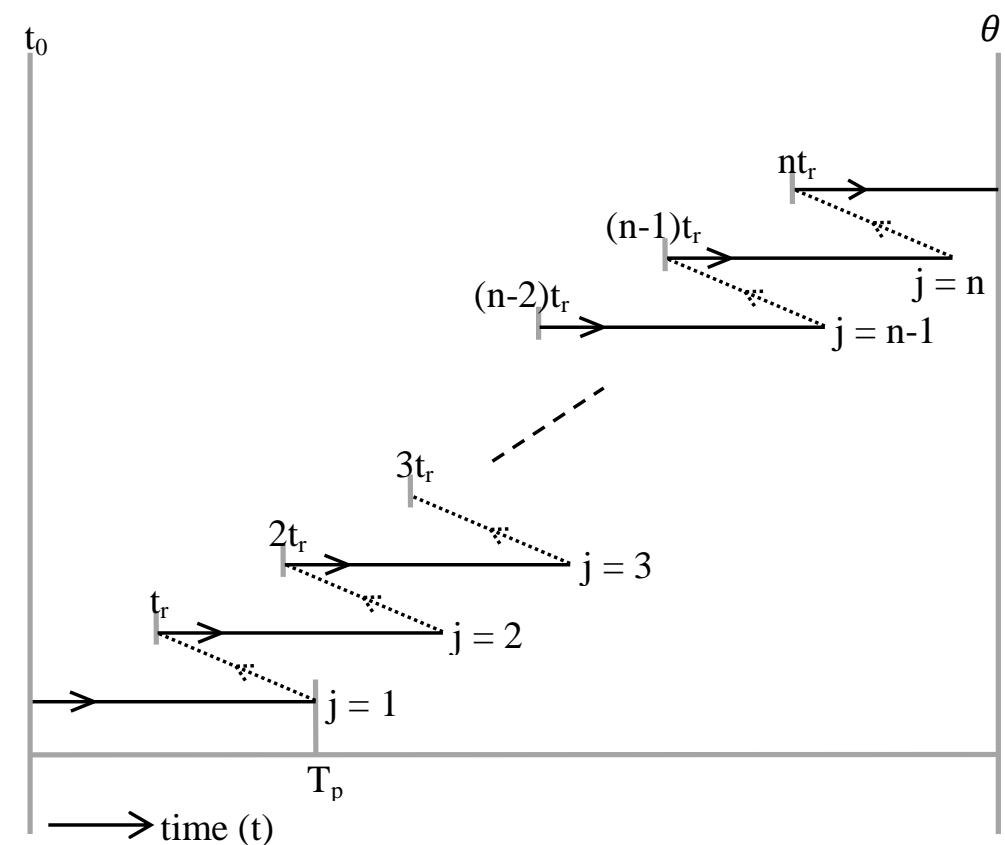

Figure 1. Universal Modeling of Reliability under IPM

Therefore, according to the principle of mathematical induction, it follows that:

$$
\begin{gathered}
R(t)=R\left(T_{p}\right) R\left(t_{r}, t_{r}+T_{p}\right) R\left(2 t_{r}, 2 t_{r}+T_{p}\right) \ldots R\left((n-2) t_{r},(n-2) t_{r}+T_{p}\right) R\left((n-1) t_{r},(n-1) t_{r}\right. \\
\left.+T_{p}\right) \quad ; j=n \wedge t=n T_{p}
\end{gathered}
$$


A further simplification of equation 4 which cannot be enumerated exhaustively here gives equation 5 as the modeling for the probability of the component surviving $n$ PM stages.

$R\left(T_{p}\right)^{n}=\prod_{j=1}^{n} R\left((j-1) t_{r},(j-1) t_{r}+T_{p}\right)$

The second aspect to be addressed according to equation 3 is the probability of the component surviving the remaining time $t_{\text {rem }}$ which is trivial from Figure 1 and is given by equation 6 .

$R\left(t_{\text {rem }}\right)=R\left(n t_{r}, \quad n t_{r}+\left(t-n T_{p}\right)\right)$

Hence the product of equations 5 and 6 gives the universal model for reliability $R_{u}(t)$ under IPM as shown in equation 7.

$R_{u}(t)=\left(\prod_{j=1}^{n} R\left((j-1) t_{r},(j-1) t_{r}+T_{p}\right)\right) R\left(n t_{r}, n t_{r}+\left(t-n T_{p}\right)\right)$

The probability of failure $\mathrm{F}(\mathrm{x}, \mathrm{y})$ between two points $\mathrm{x}$ and $\mathrm{y}$ can be given as:

$F(x, y)=F(y)-F(x) \quad ; x \leq y$

According to Márquez [5]:

$R(t)=1-F(t)$; therefore, $F(t)=1-R(t)$, substituting this in equation 8 and simplifying it gives equation 9 .

$$
R(x, y)=1-R(x)+R(y)
$$

Using equation 9 , equation 7 is transformed into 10 below, giving the universal model for reliability under IPM.

$$
\begin{aligned}
R_{u}(t)=\left(\prod_{j=1}^{n}\left(1-R\left((j-1) t_{r}\right)+R\left((j-1) t_{r}+T_{p}\right)\right)\right)\left(1-R\left(n t_{r}\right)\right. \\
\left.+R\left(n t_{r}+\left(t-n T_{p}\right)\right)\right)
\end{aligned}
$$

\subsection{Weibull Modeling of Reliability under IPM}

The reliability model without the effect of preventive maintenance under Weibull distribution is as shown in equation 11 [5]. Where $\gamma, \theta$ and $\beta$ are location, scale and shape parameter respectively.

$$
R(t)=\exp \left[-\left(\frac{t-\gamma}{\theta}\right)^{\beta}\right]
$$

The component reliability model under IPM using the Weibull distribution model can be established using the universal model (equation 10). Substituting equation 11 into 10 with further simplification of the resultant equation gives equation 12; the Weibull model for component reliability under IPM. 


$$
\begin{gathered}
R_{c}(t)=\prod_{j=1}^{n}\left(1-\exp \left[-\left(\frac{(j-1) t_{r}-\gamma}{\Theta}\right)^{\beta}\right]+\exp \left[-\left(\frac{\left((j-1) t_{r}+T_{p}\right)-\gamma}{\Theta}\right)^{\beta}\right]\right)(1 \\
\left.-\exp \left[-\left(\frac{n t_{r}-\gamma}{\Theta}\right)^{\beta}\right]+\exp \left[-\left(\frac{\left(n t_{r}+\left(t-n T_{p}\right)\right)-\gamma}{\Theta}\right)^{\beta}\right]\right)
\end{gathered}
$$

Although this paper focuses on imperfect preventive maintenance, its mathematical correctness can be checked against an existing perfect preventive maintenance model in literature which also considered component rejuvenation. The said perfect preventive maintenance model exists in [8]. As mentioned earlier, a preventive maintenance becomes perfect when the improvement factor $f=1$. This infers that if equation 12 is sufficient to modeling imperfect preventive maintenance, then it should transform to a perfect preventive maintenance model when $f=1$. Thus, when $f=1, t_{r}$ evaluates to 0 since $t_{r}=(1-f) T_{p}$, and since origin starts from 0 , i.e. when component was new, $\gamma=0$, and hence by substituting for $t_{r}$ and $\gamma$ in equation 12, the equation is mathematically transformed into the perfect preventive maintenance model found in [8], shown in equation 13 below.

$R_{c}(t)=\exp \left[-n\left(\frac{T_{p}}{\theta}\right)^{\beta}\right] \exp \left[-\left(\frac{t-n T_{p}}{\theta}\right)^{\beta}\right]$

Therefore, the transformation of the imperfect preventive maintenance model (equation 12) into a perfect preventive maintenance model (equation 13) has mathematically suggested that equation 12 is sufficient to modeling imperfect preventive maintenance under the assumptions of the proportional age reduction model and improvement factor.

\section{Case Study}

A simplified model of the fuel oil service system (FOSS) is considered as a case study to evaluating the established reliability model under IPM (equation 12). The FOSS is as seen in Figure 2. It comprises of a Service tank in which the fuel oil is stored following purification from water and debris. The Service tank has a heating coil to heat up the fuel oil and to also maintain viscosity, thereby making the fuel oil easier to be pumped. The Service tank is connected to a Booster pump which pumps fuel oil to a Mixing tank via an Automatic filter and a Flow meter. The filter further purifies the fuel oil while the Flow meter ensures flow of fuel oil to the Mixing tank.

The Mixing tank is also connected to the Service tank and this is useful in situation where the pressure in the Mixing tank exceeds a defined value. In such situation, the excess fuel oil is released into the Service tank. From the Mixing tank, fuel oil is transported to the Main engine through a Circulation pump. This pump is connected to a Heater which also makes viscosity of the fuel oil easier. The Heater in turn is connected to a Viscosimeter which regulates the working of the heater. An Indicator filter which takes its supply from the Viscosimeter further purifies the fuel oil before reaching the Main engine. Excess amount of fuel oil in the Main engine is flown back to the Mixing tank through the connecting pipe between them.

HiP-HOPS [9] a state-of-the-art dependability analysis tool was used in the reliability analysis of the FOSS. The reliability model under IPM (equation 12) was implemented in HiP-HOPS to be used as the component reliability evaluation model. Additionally, the constituent components of the FOSS were in HiP-HOPS manually annotated with their 
respective failure data, and are basically specified as list of internal failure modes of the components and a list of deviations of parameters as they can be observed at component outputs. The HiP-HOPS analysis technique was first developed by Papadopoulos and McDermid [10].

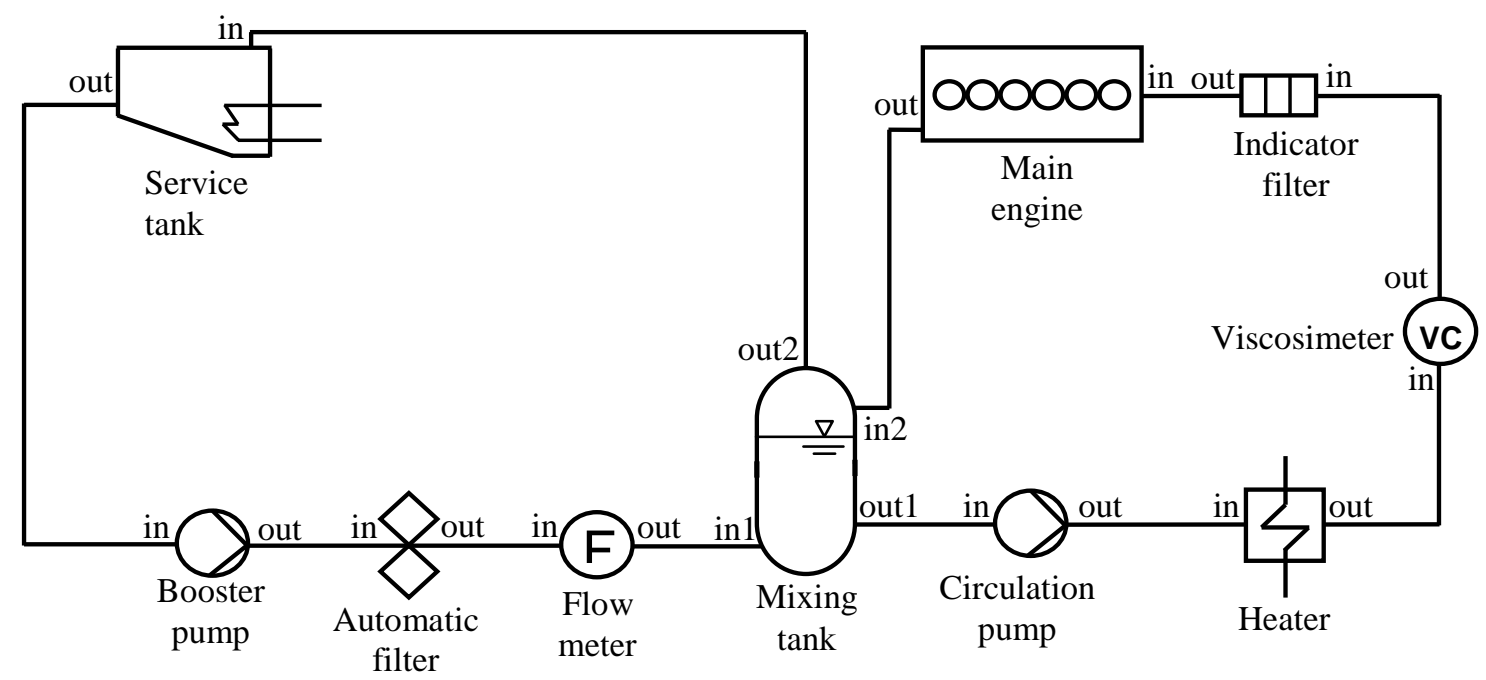

Figure 2. Fuel Oil Service System (FOSS)

\section{Results and Evaluation}

The evaluation was carried out in two forms. Firstly, under two improvement factor values $f_{1}$ and $f_{2}$, where $f_{1}<f_{2}$. As the improvement factor approaches unity, reliability is expected to improve further. Hence reliability under $f_{2}$ is expected to improve more than under $f_{1}$. Secondly, reliability is also more improved when $T_{p}$ is more frequent. Although under this scenario the cost of maintenance will be more, the evaluation of this scenario will attempt to establish that equation 12 is sufficient to modeling component reliability under IPM. The investigation of a trade-off between improved reliability and cost is left out of the scope of this paper. Component reliability under no PM is evaluated using equation 11 while that under IPM is evaluated using equation 12. The system reliability is evaluated using the EsaryProschan approximation [11] which is applied on the minimal cut sets of the fault trees produced by HiP-HOPS analysis of the FOSS model.

In both evaluations, the following parameter values were commonly used and the results are presented in appropriate sections of this paper.

Weibull scale parameter $\theta=1500$

Weibull shape parameter $\beta=2$

Weibull location parameter $\gamma=0$; i.e., when component was new

Improvement factors $f_{1}=0.565, f_{2}=0.865$ 


\subsection{Effect of Improvement Factor}

The evaluation assumes $T_{p}=120$ time units. Due to the large time scale considered and space limitation, it is not possible to exhaustively show reliability improvements from $0,1,2$, ..1499, 1500 time units. Therefore, Table 1 shows reliability evaluations at time intervals: 0 , $60,120, . ., 1440,1500$. It shows reliability evaluations under no PM policy, and that under PM policy. Additionally, the reliability under PM policy contains evaluations under two different improvement factors. In general, the results show that system reliability is improved under preventive maintenance. The reliability in all cases is same for $t \leq 120$, and this is expected since the effective age is assumed to take effect after PM stages. Also in contrast, reliability is more improved under $f_{2}$ than $f_{1}$. This characteristic is also expected since as improvement factor approaches the value 1, reliability is improved better. Hence, the effectiveness of maintenance actions plays a role in improving reliability.

Table 1. Effect of Improvement Factor on System (FOSS) Reliability

\begin{tabular}{|r|r|r|r|}
\hline & \multicolumn{3}{|c|}{ Reliability } \\
\cline { 3 - 4 } Time $(\boldsymbol{t})$ & Under No PM & \multicolumn{2}{|c|}{ Under IPM } \\
\cline { 3 - 4 } & & $f_{1}=0.565$ & $f_{2}=0.865$ \\
\hline 0 & 1 & 1 & 1 \\
\hline 60 & 0.985703 & 0.985703 & 0.985703 \\
\hline 120 & 0.944027 & 0.944027 & 0.944027 \\
\hline 180 & 0.878447 & 0.907549 & 0.923326 \\
\hline 240 & 0.794216 & 0.84774 & 0.877443 \\
\hline 300 & 0.697676 & 0.795026 & 0.851563 \\
\hline 360 & 0.595473 & 0.724528 & 0.802996 \\
\hline 420 & 0.493812 & 0.663101 & 0.773295 \\
\hline 480 & 0.397882 & 0.589847 & 0.723574 \\
\hline 540 & 0.311486 & 0.527133 & 0.691446 \\
\hline 600 & 0.236928 & 0.457986 & 0.642021 \\
\hline 660 & 0.1751 & 0.399956 & 0.608804 \\
\hline 720 & 0.125732 & 0.339686 & 0.560964 \\
\hline 780 & 0.0877205 & 0.290144 & 0.527874 \\
\hline 840 & 0.0594631 & 0.241128 & 0.482694 \\
\hline 900 & 0.0391639 & 0.20166 & 0.450764 \\
\hline 960 & 0.0250621 & 0.164183 & 0.409066 \\
\hline 1020 & 0.0155826 & 0.134604 & 0.379115 \\
\hline 1080 & 0.0094136 & 0.107501 & 0.341459 \\
\hline 1140 & 0.0055254 & 0.0865121 & 0.314078 \\
\hline 1200 & 0.00315111 & 0.0678738 & 0.280771 \\
\hline 1260 & 0.00174605 & 0.0536944 & 0.256327 \\
\hline 1320 & 0.000940029 & 0.0414479 & 0.227447 \\
\hline 1380 & 0.000491721 & 0.0322819 & 0.206106 \\
\hline 1440 & 0.000249912 & 0.0245585 & 0.181543 \\
\hline 1500 & 0.00012341 & 0.0188621 & 0.163299 \\
\hline & & & \\
\hline & & & \\
\hline & & &
\end{tabular}


To visually view reliability improvements, Figure 3 is a pictorial and detailed representation of Table 1 with time step of 1 ; i.e., $0,1,2, . ., 1499,1500$. The Green plot or $\mathrm{R}_{1}$ shows reliability with improvement factor $f_{1}$, grey plot or $\mathrm{R}_{2}$ denotes reliability with improvement factor $f_{2}$ and the black plot or $\mathrm{R}_{3}$ is reliability under no PM policy. The figure clearly shows IPM reliability improvements over no PM policy. The scale of IPM reliability improvement under two different improvement factors can be easily observed in Figure 3.

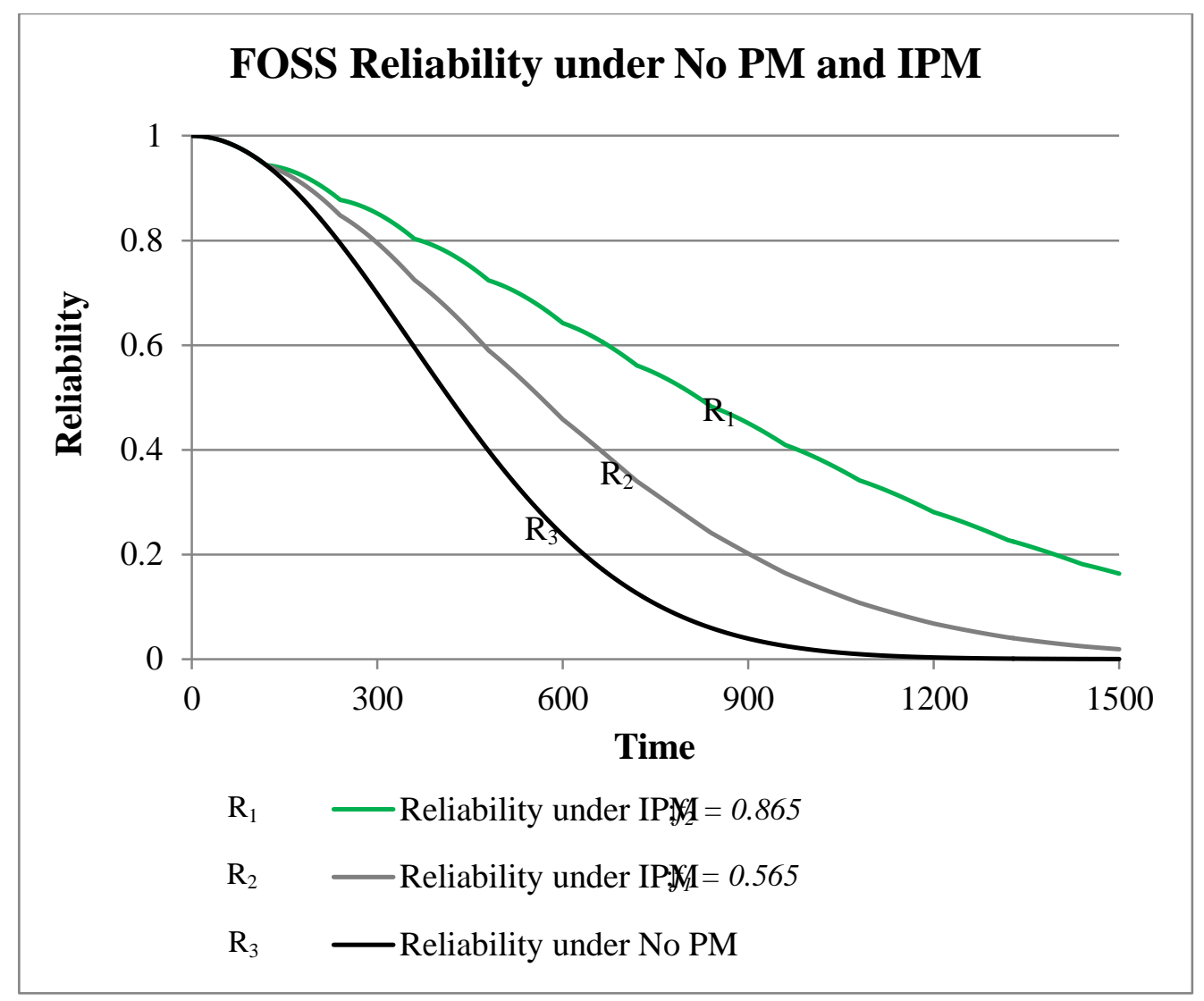

Figure 3. FOSS Reliability under no PM and IPM Policy

\subsection{Effect of PM Interval}

To investigate the effect of PM interval on system reliability, two PM intervals $T_{p l}=120$ and $T_{p 2}=180$ are considered under both improvement factors $\left(f_{1}\right.$ and $\left.f_{2}\right)$. The result is as shown in Table 2. As it is the case in Table 1, the reliability in all cases is same for $t \leq 120$. It can be observed that system reliability under both improvement factors is improved better when the PM interval is more frequent.

Table 2. Effect of PM Interval on System (FOSS) Reliability

\begin{tabular}{|r|r|r|r|r|r|r|}
\hline \multirow{2}{*}{ Time } & \multicolumn{2}{|c|}{ Reliability } & \multirow{2}{*}{} & \multicolumn{2}{|c|}{ Reliability } \\
\cline { 2 - 4 } \cline { 5 - 7 } & \multicolumn{2}{|c|}{$\mathrm{f}=0.565$} & & \multicolumn{2}{c|}{$\mathrm{f}=0.865$} \\
\cline { 2 - 5 } \cline { 5 - 7 } & $\mathrm{T}=120$ & $\mathrm{~T}=180$ & & Time & $\mathrm{T}=120$ & $\mathrm{~T}=180$ \\
\hline 0 & 1 & 1 & & 0 & 1 & 0.9857 \\
\hline 60 & 0.9857 & 0.9857 & & 60 & 0.9857 & 0.9857 \\
\hline
\end{tabular}




\begin{tabular}{|r|r|r|l|r|r|r|}
\hline 120 & 0.94403 & 0.94403 & & 120 & 0.94403 & 0.94403 \\
\hline 180 & 0.90755 & 0.87845 & & 180 & 0.92333 & 0.87845 \\
\hline 240 & 0.84774 & 0.83407 & & 240 & 0.87744 & 0.85585 \\
\hline 300 & 0.79503 & 0.76951 & & 300 & 0.85156 & 0.81017 \\
\hline 360 & 0.72453 & 0.68985 & & 360 & 0.803 & 0.74517 \\
\hline 420 & 0.6631 & 0.63136 & & 420 & 0.7733 & 0.7176 \\
\hline 480 & 0.58985 & 0.56162 & & 480 & 0.72357 & 0.67146 \\
\hline 540 & 0.52713 & 0.48556 & & 540 & 0.69145 & 0.61047 \\
\hline 600 & 0.45799 & 0.42891 & & 600 & 0.64202 & 0.58112 \\
\hline 660 & 0.39996 & 0.36839 & & 660 & 0.6088 & 0.53751 \\
\hline 720 & 0.33969 & 0.30768 & & 720 & 0.56096 & 0.4831 \\
\hline 780 & 0.29014 & 0.26281 & & 780 & 0.52787 & 0.4546 \\
\hline 840 & 0.24113 & 0.21841 & & 840 & 0.48269 & 0.41569 \\
\hline 900 & 0.20166 & 0.17662 & & 900 & 0.45076 & 0.36937 \\
\hline 960 & 0.16418 & 0.14623 & & 960 & 0.40907 & 0.34363 \\
\hline 1020 & 0.1346 & 0.11789 & & 1020 & 0.37912 & 0.31066 \\
\hline 1080 & 0.1075 & 0.09257 & & 1080 & 0.34146 & 0.27294 \\
\hline 1140 & 0.08651 & 0.0745 & & 1140 & 0.31408 & 0.25106 \\
\hline 1200 & 0.06787 & 0.05845 & & 1200 & 0.28077 & 0.22443 \\
\hline 1260 & 0.05369 & 0.04472 & & 1260 & 0.25633 & 0.19499 \\
\hline 1320 & 0.04145 & 0.03509 & & 1320 & 0.22745 & 0.17735 \\
\hline 1380 & 0.03228 & 0.02689 & & 1380 & 0.20611 & 0.15679 \\
\hline 1440 & 0.02456 & 0.02012 & & 1440 & 0.18154 & 0.13473 \\
\hline 1500 & 0.01886 & 0.01545 & & 1500 & 0.1633 & 0.1212 \\
\hline
\end{tabular}

The graphical presentations of the detailed results of Table 2 are as shown in Figures 4 and 5. Figure 4 shows the system reliability under the two different PM intervals $T_{p 1}$ and $T_{p 2}$ using improvement factor $f_{l}$. Figure 5 also shows the system reliability under the two different PM intervals but both using improvement factor $f_{2}$. The improvement in system reliability as earlier mentioned with regards to Table 2 can clearly be seen in Figures 4 and 5. 


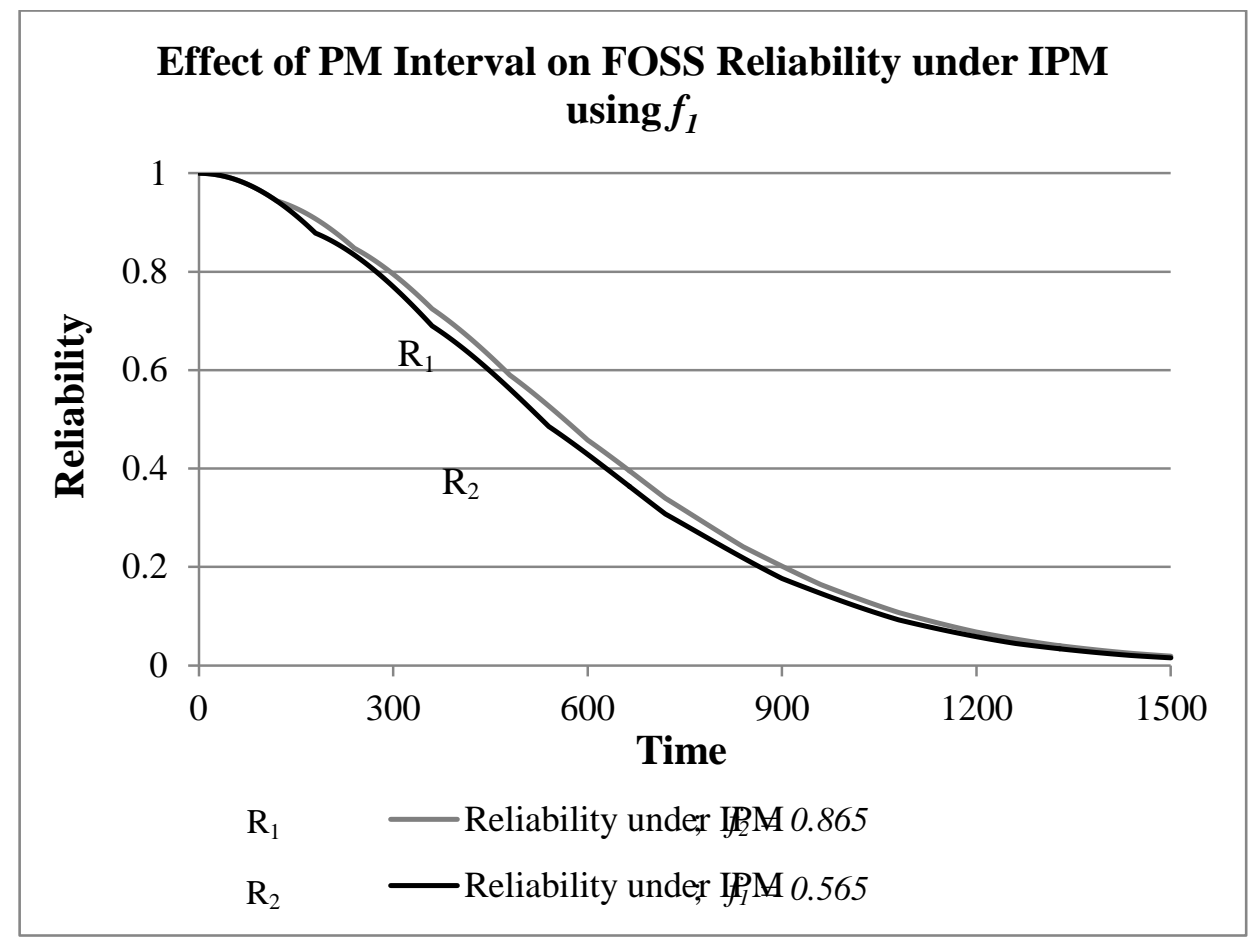

Figure 4 - Effect of PM Interval on System (FOSS) Reliability Using $f_{1}$

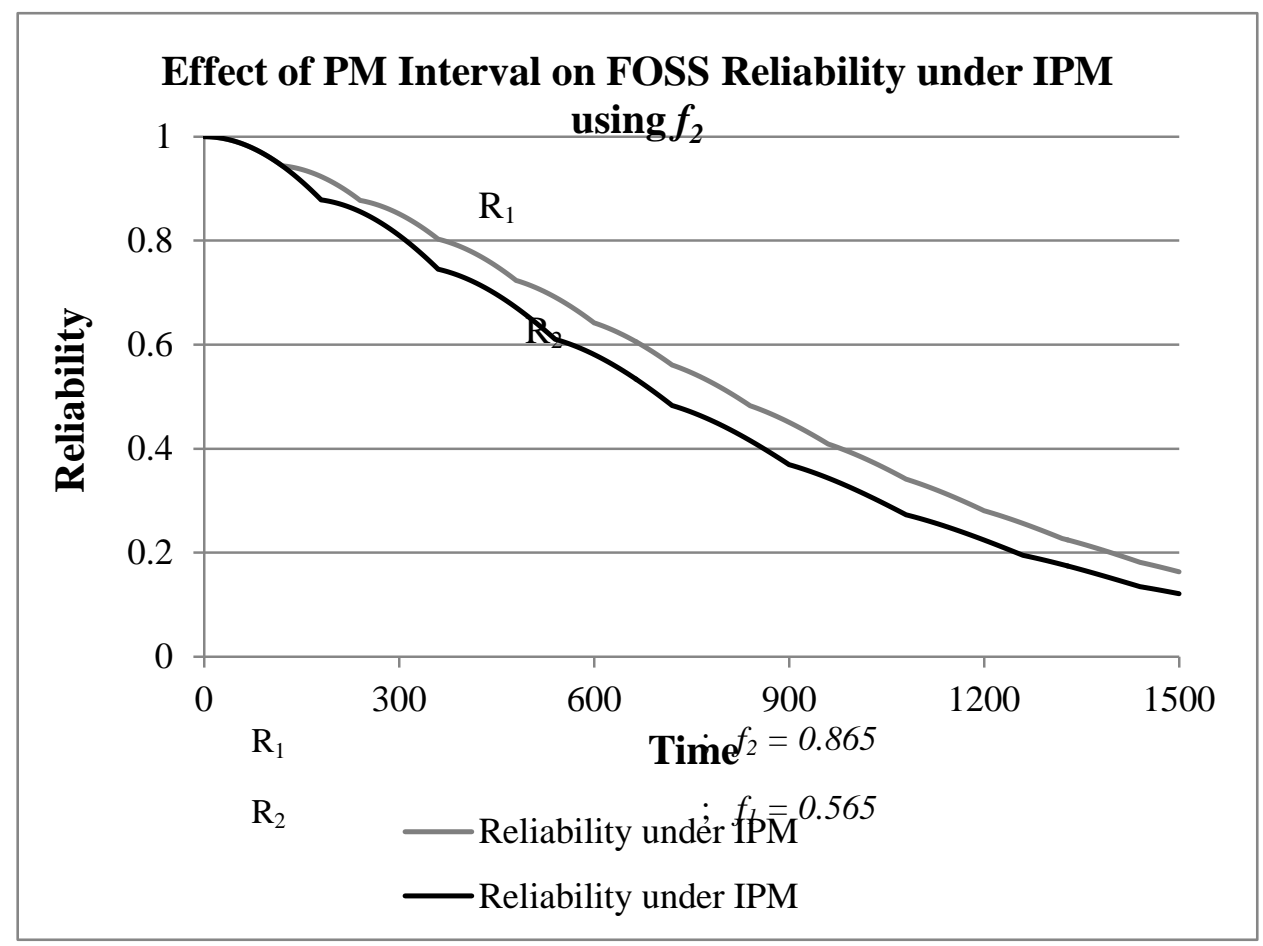

Figure 5. Effect of PM Interval on System (FOSS) Reliability Using $f_{2}$ 


\section{Conclusions}

This paper has investigated the effect of imperfect preventive maintenance (IPM) on component reliability. The proportional age reduction (PAR) model was considered together with the concept of improvement factor. A universal model for component reliability is established with which further modeling could be performed with respect to a given failure distribution. In particular, this paper assumed that component failure characteristics follow the Weibull distribution. As a result, a novel model for component reliability under IPM was established. To evaluate the model, the fuel oil service system (FOSS) which supplies oil to the main engine of a container ship was considered as case study. Typically, system reliability is influenced by the reliabilities of its constituent components. Therefore components reliability under IPM were evaluated using the established model in this paper while system reliability was evaluated using the Esary-Proschan approximation. Relative to a no preventive maintenance policy, the results obtained show that (i) system reliability is improved under IPM, (ii) as the value of the improvement factor approaches 1 , system reliability is improved better and, (iii) that system reliability is improved better when the preventive maintenance interval is more frequent. However, frequent maintenance may be expensive in terms of maintenance cost and it is usually solvable through optimisation which is out of the scope of this paper.

The results obtained are typically the expected behavioural patterns of a system under a given preventive maintenance policy. Thus the results suggest that the established reliability model is sufficient to modeling component reliability under imperfect preventive maintenance policy using the proportional age reduction model.

\section{References}

[1] Y. Lin, J. Chen and Y. Chen, "The Impact of Inspection Errors, Imperfect Maintenance and Minimal Repairs on an Imperfect Production System”, Mathematical and Computer Modelling, vol. 53, no. 9-10, (2011), pp. 1680-1691.

[2] K. S. Moghaddam and J. S. Usher, "Preventive Maintenance and Replacement Scheduling for Repairable and Maintainable Systems using Dynamic Programming”, Computers \& Industrial Engineering, vol. 60, no. 4, (2011), pp. 654-665.

[3] S. Martorell, A. Sanchez and V. Serradell, "Age-Dependent Reliability Model Considering Effects of Maintenance and Working Conditions”, Reliability Engineering and System Safety, vol. 64, no. 1, (1999), pp. 19-31.

[4] Y. Tsai, K. Wang and L. Tsai, "A Study of Availability-Centered Preventive Maintenance for MultiComponent Systems", Reliability Engineering and System Safety, vol. 84, no. 3, (2004), pp. 261-270.

[5] A. C. Márquez, "The Maintenance Management Framework: Models and Methods for Complex Systems Maintenance", Springer Series in Reliability Engineering, (2007).

[6] Y. Tsai, K. Wang and H. Teng, "Optimizing Preventive Maintenance for Mechanical Components using Genetic Algorithms", Reliability Engineering and System Safety, vol. 74, no. 1, (2001), pp. 89-97.

[7] A. Sanchez, S. Carlos, S. Martorell and J. F. Villanueva, "Addressing Imperfect Maintenance Modelling Uncertainty in Unavailability and Cost Based Optimization”, Reliability Engineering and System Safety, vol. 94, no. 1, (2009), pp. 23-32.

[8] C. E. Ebeling, “An Introduction to Reliability and Maintainability Engineering”, McGraw-Hill, USA, (1997).

[9] S. H. Nggada, D. J. Parker and Y. I. Papadopoulos, "Dynamic Effect of Perfect Preventive Maintenance on System Reliability and Cost Using HiP-HOPS", IFAC-MCPL 2010, 5th Conference on Management and Control of Production and Logistics, Coimbra, Portugal, (2010) September.

[10] Y. I. Papadopoulos and J. A. McDermid, "Hierarchically Performed Hazard Origin and Propagation Studies, 18th International Conference in Computer Safety, Reliability and Security, Toulouse, France, (1999).

[11] T. Jin and D. W. Coit, "Approximating Network Reliability Estimates using Linear and Quadratic Unreliability of Minimal Cuts”, Reliability Engineering and System Safety, vol. 82, no. 1, (2003), pp41-48. 


\section{Author}

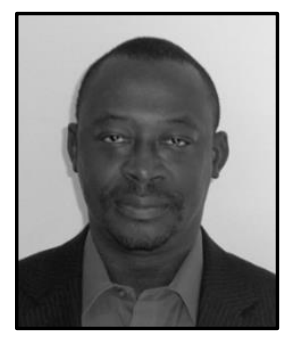

Shawulu Hunira Nggada has PhD in Computer Science and PGDp in Research Training both from University of Hull - UK, MSc Software Engineering from University of Bradford - UK and B. Tech (Hons) in Computer Science from Abubakar Tafawa Balewa University, Nigeria. $\mathrm{He}$ is a Charted IT Professional with BCS The Charted Institute for IT, Member Nigeria Computer Society and IEEE Reliability Society. He is currently an Associate Professor in Computer Science at the Polytechnic of Namibia (transforming into: Namibia University of Science and Technology). 